\title{
Objetos de Aprendizagem voltados ạo ensino de modelagem digital 30 para arquitetura baseado no estilo cognitivo visual
}

\author{
Learning Objects directed to teaching digital 30 modeling for architecture based on visual cognitive style
}

\author{
> Rafael Zanelato Ledo \\ Universidade Estadual de Santa Catarina, Brasil \\ rafael.ledo@udesc.br
}

\author{
> Alice Theresinha Cybis Pereira \\ Universidade Federal de Santa Catarina, Brasil \\ acybis@gmail.com
}

\begin{abstract}
This article describes an initiative to identify which cognitive abilities influence performance in 3D modeling for architecture activities. This work tests the visual, spatial and logical reasoning skills of students of architecture. After these tests, the results will compared with the results of $3 \mathrm{D}$ modeling test. By identifying, some cognitive abilities that has a direct relation to performance in 3D modeling will be possible to create learning objects that improve these characteristics.
\end{abstract}

Keywords: 3D Modeling, Cognitive Syles, Visuospatial Thinking, Performance, Architecture

\section{Introdução}

Este artigo apresenta uma pesquisa de Doutorado em desenvolvimento que questiona os objetos de aprendizagem existentes para o ensino de modelagem digital $3 \mathrm{D}$ voltado à arquitetura. O questionamento não é sobre a quantidade de objetos de aprendizagem existentes ou sobre quais softwares estes estes objetos trabalham, mas sim a sua adaptação ao modo de processar a informação de cada indivíduo. Para isso poder ser feito, antes é preciso produzir uma pesquisa que identifique quais aspectos cognitivos influenciam a atividade de modelar em 3D para Arquitetura através dos programas computacionais. Este é o objetivo deste trabalho. Esta investigação utiliza como base as teorias e métodos científicos da Psicologia Cognitiva.

$\mathrm{Na}$ Psicologia Cognitiva há duas linhas de pesquisa que se diferenciam que são a pesquisa experimental e a pesquisa correlacional. A pesquisa experimental está direcionada a estudar isoladamente as atividades mentais como a memória de trabalho, memória de longo termo, atenção, raciocínio lógico entre outros e o que influencia e como ocorrem estas atividades. Em contrapartida a pesquisa correlacional como descreve Hegarty \& Waller (2005), permite através da comparaçáo entre indivíduos estabelecer para determinada atividade o quanto uma habilidade é importante. Por exemplo, para a atividade de resoluçáo de problemas matemáticos possuir alta habilidade espacial influencia ou não o desempenho?

É dentro deste contexto que este artigo estabelece sua questão de pesquisa. Quais habilidades cognitivas influenciam o desempenho durante a atividade de modelagem digital $3 \mathrm{D}$ para arquitetura? $\mathrm{O}$ método que buscará essas respostas é a correlação. Isso se dará através de testes com estudantes de Arquitetura no quais os resultados da avaliação cognitiva serão confrontados com o desempenho na modelagem 3D.

A resposta a questão de pesquisa pode levar diversas outras investigaçóes ou aplicaçóes. Este trabalho cita três situaçóes como; o aperfeiçoamento das interfaces dos softwares 3D tornando-as adaptativas as habilidades cognitivas dos usuários, a seleção para o emprego e o foco principal deste trabalho que é a criaçáo de objetos de aprendizagem que reforcem e desenvolvam as habilidades cognitivas necessárias para um melhor desempenho na atividade de modelagem digital 3D.

\section{A atividade de modelagem $3 D$ para Arquitetura}

O uso da informática na Arquitetura já existe há décadas e muito do que foi produzido em termos de Arquitetura Contemporânea está relacionada ao desenvolvimento da Computaçáo Gráfica. Diversos autores como Portella (2006) e Kalay (2006) possuem trabalhos discutindo o impacto da Computação Gráfica no processo de projeto, na prototipagem e no resultado final da edificação produzida.

O que se observa em todos os trabalhos é que o modelo digital 3D desempenha um papel central no processo contemporâneo do desenvolvimento do projeto de arquitetura. Esta centralidade têm ganhado ainda mais força com a inclusão das informaçóes de projeto dentro da estrutura de dados do modelo 3D definindo a essência do BIM , (Building Information Modeling ). 
Atualmente é a partir das informaçóes contidas e geradas a partir do modelo digital 3D que se desenvolve o projeto arquitetônico através da verificação de sua Performance Bioclimática geração de Quantitativos/Orçamentos e Compatibilização dos projetos das diversas disciplinas. Também é através do modelo 3D que se desenvolve a representação do projeto através da geração de imagens/ animaçōes fotorealísticas, do desenho técnico arquitetônico através da geração das projeções bidimensionais e da maquete física. E por fim têm-se a própria edificação que pode ter seus componentes construídos a partir do modelo digital 3D no sistema CAM (Computer-Aided Manufacturing).

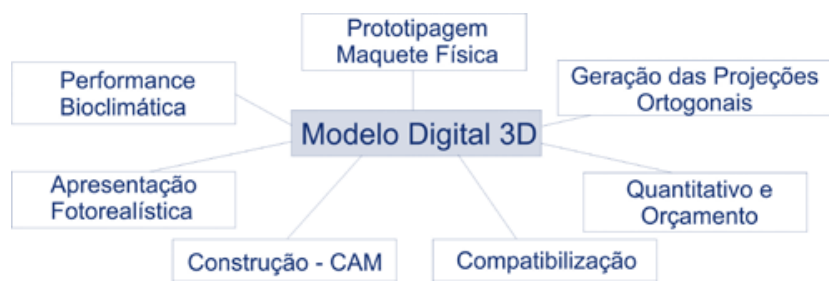

Figura 1: Atividades derivadas do Modelo Digital 3D de Arquitetura. Fonte: autores.

A evidente importância da participação do modelo digital 3D na Arquitetura da atualidade traz a necessidade de se aumentar e aperfeiçoar o aprendizado da competência - modelagem 3D na formação dos futuros arquitetos. $\mathrm{O}$ avanço desta competência trará maior desempenho nessa atividade possibilitando a criação de modelos cada vez mais precisos e complexos e cada vez num tempo menor.

A resolução de problema de projeto versus problema de modelagem.

A modelagem digital 3D de Arquitetura pode ocorrer em diferentes etapas do processo de projeto. Ela pode estar presente no processo inicial de criaçáo durante a etapa de Estudo Preliminar ou no final do processo de projeto com intuito de fazer uma apresentação de como irá ficar a edificação ao cliente.

Essa diferenciação da etapa na qual ocorre o uso da modelagem digital 3D é importante porque o processo de geração 3D é diferenciado. Quando a modelagem 3D é feita nas etapas iniciais do projeto ainda não há uma forma definida. O uso da ferramenta computacional é no intuito de gerar formas que atendam a uma série de requisitos projetuais. Quando a modelagem é feita ao final do processo não há um processo criativo em curso em termos de desenvolvimento de projeto. Só há a transformação da idéia num modelo 3D.

Essa abordagem tem que ser destacada pois tanto o ato de projetar quanto o de modelar envolvem a resolução de problema, cada um com suas próprias características. Com o projeto já definido o único problema é como modelar em 3D. Quais comandos e açôes utilizar num determinado software para alcançar uma forma já determinada? Quando se está projetando há dois problemas a resolver, o próprio projeto em si e como modelar a forma que estamos imaginando para atender os requisitos existentes.

\section{Habilidades Cognitivas}

Quais habilidades cognitivas mais influenciam o desempenho na resolução do problema da modelagem 3D? E quais essas habilidades quando este problema envolve, além da modelagem, decisóes de projeto?

O modelo de intelecto não possui somente uma ou outra dimensão como a espacial, quantidade de memória ou raciocínio lógico, mas sim várias subdivisóes. Somente no Manual de testes de aptidão organizado por Elkstron et al (1976) são avaliados 23 fatores cognitivos. Para analisar aspectos cognitivos de estudantes que desenvolveriam um trabalho de modelagem $3 \mathrm{D}$ optou-se pelos fatores mais evidentes a essa atividade. O primeiro deles foi o pensamento visuopespacial, depois a memória visual e por fim o raciocínio lógico.

\section{Habilidade Visuoespacial}

A habilidade visuoespacial na Psicologia Cognitiva é a habilidade de entender como objetos rotacionam e se movimentam no espaço e suas possíveis transformações. São denominadas de imagens mentais. Estas imagens mentais nos permitem relembrar objetos e situaçóes que foram vistos algum tempo ou imaginar e criar objetos que nunca foram observados. (Matlin, 2003; Sternberg,2008)

Quando se investiga as imagens mentais duas abordagens bastante explorada pelos pesquisadores devem ser consideradas para o entendimento deste fenômeno. A primeira abordagem é que existem duas teorias antagônicas que explicam a formação das imagens mentais que são; o código analógico e o código propositivo.

O código analógico diz que as imagens mentais que se produz são fiéis a percepção que se faz de uma determinada figura. Pesquisa de Shepard e Meltzer (1971) demonstra que o tempo que demora-se para imaginar um objeto rotacionar no espaço é proporcional ao que teria se tivesse sendo rotacionado com os as máos. $O$ código propositivo declara que o armazenamento de imagens mentais se dá através de elementos simbólicos que descrevem uma relação visual e espacial. Estes códigos são ativados antes do cérebro organizar a imagem. Para ilustrar o código propositivo faz-se uso do cálculo de predicados utilizado em lógica.

Embora a teoria mais aceita seja a do código analógico em decorrência da quantidade de testes empíricos confirmatórios que foram realizados, o código propositivo também é considerado pois o analógico não consegue explicar todas as situaçóes (Sternberg,2008). Pesquisadores que defendem o código propositivo como Pylyshyn, (2001) argumentam que se só houvesse o código analógico não teríamos espaço suficiente na memória para armazenar todas as imagens mentais.

Estas teorias deixam alguns questionamentos quando se pensa em modelagem 3D. Quando se modela uma forma já conhecida, numa situação de resolução de problema de modelagem quais códigos usamos? Os dois ou um prevalece? E quando é uma situaçấo no qual a forma é desconhecida que envolve a resolução do problema de projeto também, quais códigos usamos e em qual intensidade?

A segunda abordagem é a teoria do What-Where systems. Essa teoria determina que o caminho do visual e do espacial são distintos no cérebro. Entende-se como visual todo estimulo relacionado a 
forma, cor ou brilho enquanto espacial relacionado a distâncias, orientaçôes e profundidade. Esta teoria foi alicerçada por teste com macacos de Ungerleider \& Mishkin,(1982) que mostrou o caminho ventral/temporal para o visual e o caminho parietal/dorsal para o espacial (Fig. 2). A pesquisade Farah et al (1988) ratifica algumas descobertas nesse sentido ao mostrar que lesóes cerebrais deixavam pessoas incapacitadas para atividades visuais, mas ainda aptas para atividades espaciais.

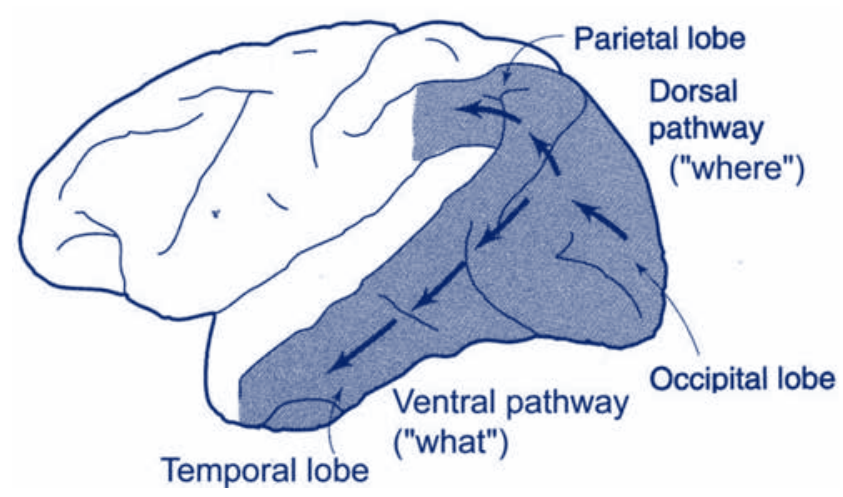

Figura 2: Caminhos ventral e dorsal definindo o caminho visual e espacial. Fonte: Adaptado de Halpern \& Coller, 2005.

\section{Estilo Cognitivo Visual}

Como descrito anteriormente, muitas pesquisas na área de Psicologia Cognitiva trabalham as diferenças individuais focando estudo correlacional. Uma área que explora esta vertente é os denominados Estilos Cognitivos. Esta vertente descreve como o indivíduo pensa, percebe, processa e resgata informação. Sempre as pessoas são classificadas dentro de uma categoria dentro dos Estilos Cognitivos. Elas podem ser definidas como Campo Dependente ou Campo Independente ou Inovadores ou Adaptadores como descritas no trabalho de Kirton (1976).

No que se refere ao pensamento visuoespacial a categorização na área sempre foi entre Verbal-Visual, pessoas que tendem a ser mais verbais e outras que tendem a ser mais visuais. Mas a pesquisa de Kozhenikov et al (2005) mostra que na realidade têm-se os verbais, mas os visuais são divididos em dois grupos, os visualizadores objeto e os visualizadores espaciais. Os visualizadores objeto são pessoas que têm mais facilidade com atividades de memorização de figuras aonde o que prevalece é a forma, cor. Os visualizadores objeto tendem a considerar a representação de modo global como uma única unidade. Os visualizadores espaciais tendem a ter mais facilidade com atividades que envolvam rotação mental e organizar elementos no espaço. Os visualizadores espaciais tendem a ter uma visão analítica se atendo a combinação das partes dos elementos.

Os estilos cognitivos visuais estão diretamente relacionados com a teoria do What-Where System, descrevendo que há para algumas pessoas o uso predominante de um dos caminhos ventral ou dorsal. Isso explicaria a diferença de desempenho nos testes de avaliação cognitiva como a Rotação Mental de Shepard \& Meltzer (1971) ou o teste Object Location Memory de Silverman \& Eals (1992). As pesquisas que demonstram as diferenças de desempenho entre homens e mulheres trilham muitas vezes o mesmo caminho. Estes testes mostram que homens têm mais facilidade com testes espaciais e as mulheres os testes de memória visual (Helpern \& Coaller,,2005).

Ao se pensar na modelagem $3 \mathrm{D}$ confrontando com as teorias descritas têm-se os questionamentos; As pessoas definidas como visualizadores espaciais possuem melhor desempenho em atividades de modelagem 3D? As mulheres possuem pior desempenho em atividades de modelagem $3 \mathrm{D}$ por não irem tão bem em atividades que envolvem a espacialidade?

\section{Habilidade Raciocínio Lógico}

A habilidade de raciocínio lógico está intimamente ligada a uma atividade de resolução de problemas que pode ser para resolver um problema de modelagem ou um problema de projeto. No caso do raciocínio a Psicologia Cognitiva destaca-se a classificação entre Dedutivo e Indutivo. O raciocínio Dedutivo está ligado a uma relação causal, aonde atendido um requisito algo logicamente ocorre, estabelecendo uma relação condicional (Sternberg, 2008)

Em modelagem 3D é muito importante possuir a estruturação de um modelo mental que tenha clareza de como os comandos funcionam e como o software responde a determinadas açóes. Dentro do raciocínio Dedutivo destaca-se também a importância do Silogismo Linear na qual retira-se uma conclusão e a partir de duas premissas e o Silogismo Categórico que possui a mesma base do Silogismo Linear mas trabalha com agrupamentos. Pode-se fazer uma relação teórica entre o Silogismo Categórico e os atuais sistemas de modelagem em BIM. A grande hierarquização de componentes presentes nas modelagens em BIM exige uma compreensão bem clara de uma estrutura sistêmica que o Silogismo Categórico também exige.

O Raciocínio Indutivo não trabalha com a certeza do Dedutivo mas com a possibilidade de algo irá acontecer ou está acontecendo a partir das observaçôes feitas. É como se estabelece o estudo empírico. $\mathrm{Na}$ modelagem muitas vezes é preciso se utilizar da indução para inferir porque certo procedimento não ocorre.

Dentro do contexto deste trabalho alguns questionamentos surgem; Pessoas que possuem alto desempenho em testes de lógica necessariamente têm melhor desempenho em testes de modelagem com o objeto final definido? E com o objeto final indefinido?

\section{Metodologia}

Para buscar responder a pergunta principal desta pesquisa que é quais aspectos cognitivos influenciam o desempenho na atividade de modelagem em $3 \mathrm{D}$ ? e as perguntas secundárias que estão postas ao longo do texto foi estruturado o procedimento metodológico.

Este procedimento está divido em duas etapas. A primeira é a Avaliação Cognitiva na qual se avalia o desempenho dos estudantes de Arquitetura em 5 fatores (Rotação Mental, Orientação Espacial, Transformação Mental, Memória Visual e Raciocínio Lógico). Há 3 agrupamentos sendo avaliados; o Espacial (Rotação Mental, Orientação Espacial, Transformação Espacial) o Visual (Memórial Visual) e Raciocínio Lógico (possui 03 testes de Raciocínio).

Foi utilizado mais de um fator para o agrupamento Espacialidade por alguns autores identificarem mais de uma fator para habilidade espacial (Hegarty \& Waller ,2005). 


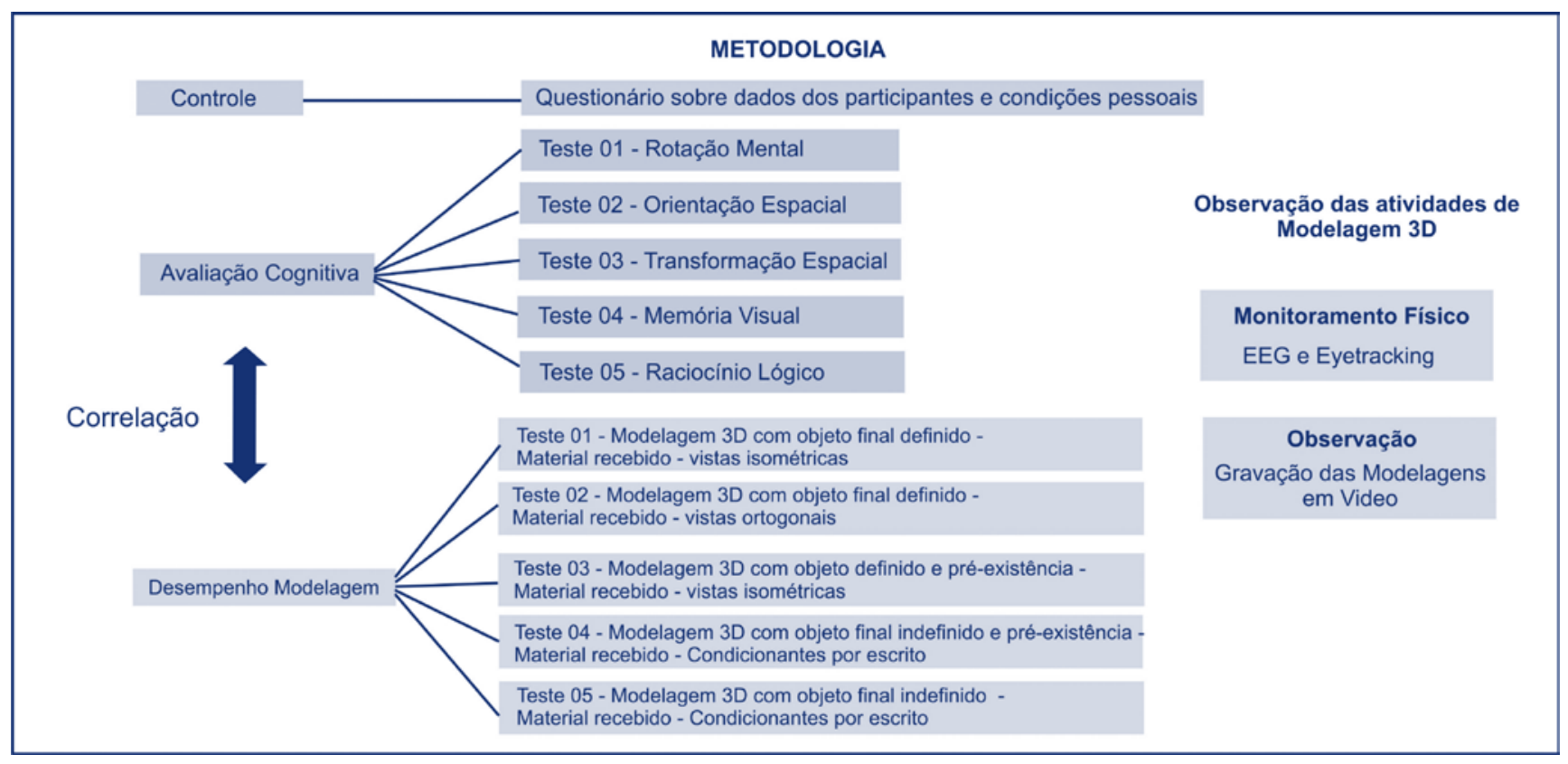

Figura Э: Quadro ilustrativo descrevendo o modelo de correlaçấo.

A segunda etapa consiste em avaliar os alunos em testes de modelagem 3D para arquitetura. Cabe destacar o que se considera desempenho nesta pesquisa para a atividade de modelagem $3 \mathrm{D}$ para Arquitetura. Desempenho nessa pesquisa é conseguir modelar de forma precisa uma edificaçáo num software 3D sem erros, no menor tempo possível utilizando-se do caminho mais curto (número de passos ou açôes) para se chegar a forma final.

Os 5 testes de modelagem simulam situaçóes de modelagem usuais que vão verificar a interferência:

Teste 01: É dado 4 vistas isométricas cotadas de uma edificação para ser modelada. Essa situação é a mais simples dos 5 testes não exigindo muito do usuário

Teste 02: É dado 4 vistas ortogonais cotadas de uma edificação a ser modelada. Esta situação exigirá boa visão espacial para montar em 3D na mente a edificação e modelar

Teste 03: É dado 4 vistas isométricas cotadas de uma edificação a ser modelada. Neste teste já há um modelo previamente modelado dentro do arquivo que deve ser transformado. Situação de reforma na arquitetura, na qual se trabalha com uma pré-existência

Teste 04: É dado somente condicionantes de projeto. Forma final indefinida. Neste teste já uma pré-existência. Tanto neste quanto no anterior se verifica a capacidade de transformação mental

Teste 05 - Neste é dado somente as condicionantes. Não há pré existência de edificação, somente um lote. Neste teste como no anterior há um problema projetual envolvido junto com o problema de modelagem.

\section{Redução de Viés}

Esta pesquisa é muito suscetível ao viés levando a coleta de dados que podem levar a conclusóes erradas. Para diminuir o viés algumas açóes seráo realizadas.
A primeira é fazer testes fisiológicos durante os testes de modelagem que serão o EEG (Eletroencefalograma) e o Eyetracking (que monitora o movimento dos olhos na tela). O teste fisiológico reduz o que os pesquisadores em Psicologia Cognitiva chamam de características de demanda, que é a influência existente fazer com que os pesquisados atingirem o resultado esperado pelo pesquisador. As modelagens serão gravadas em vídeo através da captura de tela para ser comparadas ao EEG.

A segunda ação é utilizar um software 3D que seja simples, sem grandes quantidades de comandos e recursos de forma que as diferenças no teste de modelagem não sejam necessariamente conhecimento da ferramenta. Será utilizado o Trimble Sketchup.

A terceira ação é um questionário de perfil que além dos dados básico como idade e gênero pergunta sobre motivação, cansaço, experiência com o software e tempo de faculdade.

\section{Resultados}

Nesta etapa do Doutorado o procedimento metodológico está em fase de pré-teste no qual o instrumento está sendo ajustado. Por isso a amostra é pequena, somente 10 alunos (04 mulheres e 06 homens).

$\mathrm{Na}$ Avaliação Cognitiva foi utilizado Purdue Test de Guay (1977), para os testes espaciais de Rotação Mental, Orientacão Espacial e Transformaçáo Espacial. Para Memória Visual, foi usado o Object Location Memory de Silverman \& Eals (1992) e para Raciocínio Lógico, testes RL1, RL2 e RL4 de Elkstrom (1976).

Os resultados iniciais demonstram uma correlação muito forte entre Rotação Mental e Orientação Espacial o que leva a crer em atributos que variam conjuntamente. Em se mantendo a tendência em testes posteriores pode-se eliminar um deles. Essa variação conjunta já não aparece na Transformação Mental. No teste de Memória Visual duas das quatro mulheres tiveram ótimo desempenho enquanto dos seis homens somente um teve um ótimo desempenho. Apesar de amostra pequena para se fazer inferência 
confirma o que diz na literatura. Os testes lógicos apresentaram baixo desempenho no geral de todos o que pode indicar prevalência dos aspectos visuais em detrimento da lógica em cursos de arquitetura. Esta Avaliação Cognitiva sofrerá um reteste visando a confirmação de um padrão de desempenho em cada indivíduo.

\section{Agradecimentos}

Gostaria de agradecer a Capes pelo Bolsa de Doutorado para desenvolvimento dessa pesquisa e o apoio do Laboratório de Psicologia do Esporte e Exercício da UFSC no auxílio na condução da avaliação cognitiva e na realização da avaliação neuropsicológica.

\section{Referências}

Ekstrom, R. B., French, J. W., Harman, H. H., \& Dermen, D. (1976). Kit of Factor Referenced Cognitive Tests. Princeton, NJ: Educational Testing Service.

Farah, M. J., Hammond, K. M., Levine, D. N., \& Calvanio, R. (1988). Visual and spatial memory: Dissociable systems of representation. Cognitive Psychology, 20,439-462.

Halpern, DF, \& Collaer, ML (in press). Sex differences in visuospatial abilities: More than meets the eye. In P. Shah \& A. Miyake (Eds.), Handbook of visuospatial thinking. New York: Cambridge University Press

Hegarty, Mary., \& Waller, David A.. (2005). Individual differences in spatial abilities. In P. Shah, \& A. Miyake (Eds.), The Cambridge handbook of visuospatial thinking (pp. 121-169)

Kalay, Yeshuda. (2006), The impact of information technology on design methods, products and practices, Design Studies vol. $27: 357-380$
Kirton, M. Adaptors and innovators: a description and measure, Journal of Applied Psychology (61:5) 1976, pp 622-629

Kozhevnikov, M., Kosslyn, S., \& Shephard, J. (2005). Spatial versus object visualizers: A newcharacterization of visual cognitive style. Memory \& Cognition, 33, 710-726

Matlin, Margaret. (2004) Psicologia cognitiva 5. ed. Rio de Janeiro (RJ): LTC,. 403p

Portella, Underléa Bruscato.(2006) De lo digital en arquitectura. Tese de Doutorado. Universidad Politecnica de Cataluña, Barcelona,

Guay, R. B. (1977). Purdue spatial visualization tests. West Lafayette, IN: Purdue Research Foundation.

Pylyshyn, Z. (2001). Is the imagery debate over? If so, what was it about? In E. Dupoux (Ed.), Language, brain, and cognitive development: Essays in honor of Jacques Mehler (pp. 59-83). Cambridge, MA: MIT Press.

Silverman, I., \& Eals, M. (1992). Differences in spatial abilities: Evolutionary theory and data. In J. H. Barkow, L. Cosmides, \& J. Tooby (Eds.), The adapted mind: Evolutionary psychology and the generation of culture (pp. 533-549). New York: Oxford University Press.

Sternberg, Robert J. (2010) Psicologia cognitiva. 5 ed. São Paulo: Cengage Learning,. 584p

Shepard, R. N., \& Metzler, J. (1971). Mental rotation of threedimensional objects. Science, 191, 952-954.

Ungerleider, L. G., \& Mishkin, M. (1982). Two cortical visual systems. In D. J. Ingle, R. J.W. Mansfield, \& M. S. Goodale (Eds.), The analysis of visual behavior (pp. 549-586). Cambri 